(P. Gli articoli di questa sezione sono sottoposti a referaggio doppiamente cieco (double blind peer review process) e seguono gli standard in uso per le pubblicazioni scientifiche a livello internazionale ed accettati dalle principali banche dati citazionali

\title{
Position paper \\ Per un programma di eliminazione della Epatite $C$ nella popolazione a rischio dei consumatori di sostanze e dei detenuti
}

\author{
Felice A. Nava*, Alfredo Alberti ${ }^{\circ}$, Massimo Andreoni $\$$, Sergio Babudieri^, Giorgio Barbarini**, \\ Pietro Fausto D’Egidio ${ }^{\circledR}$, Claudio Leonardi`, Alfio Lucchini ${ }^{\#}$
}

\begin{abstract}
SUMMARY
- The data recently published in scientific literature identify in substance abuse the most important risk factor for the transmission of $\mathrm{HCV}$. Another population at risk is represented by detainees, mainly because most of them have a history of substance use.

Treatment of the population at risk (substance users and detainees) must become a priority for health systems both to ensure fairness of access to care and to achieve the public health goal of eliminating $\mathrm{HCV}$.

The programs to take charge should be multi-disciplinary, flexible, tailored, evidence-based, disseminated homogeneously throughout the national territory, and supported by procedures and guidelines including harm reduction actions, as suggested by the WHO.
\end{abstract}

Keywords: Hepatitis C virus, HCV, Infection, Drugs, Detainees.

Parole chiave: Epatite C, HCV, Infezione, Sostanze, Detenuti.

\section{Introduzione}

I dati contenuti nella letteratura scientifica di recente pubblicazione individuano nel consumo di sostanze (in particolare se endovenoso) il fattore di rischio più importante per la trasmissione di HCV evidenziando altresì come i dipendenti da sostanze rappresentino il serbatoio più importante della malattia. Un'altra popolazione a rischio è rappresentata dai detenuti, soprattutto

* Direttore U.O. Sanità Penitenziaria, Azienda ULSS 6 Euganea, Padova, Direttore Comitato Scientifico FeDerSerD.

- Professore Ordinario di Gastroenterologia, Università di Padova.

$\S$ Professore Ordinario di Malattie Infettive, Università degli Studi di

"Tor Vergata” Roma.

${ }^{\wedge}$ Direttore Clinica Malattie Infettive e Tropicali, Università di Sassari, Presidente Onorario SIMSPe.

** Clinica Malattie Infettive e Tropicali Fondazione IRCCS San Matteo, Pavia, Ufficio di Presidenza CLEO.

${ }^{\circledR}$ Internista, Presidente Nazionale FeDerSerD.

- Direttore U.O.C. Patologie da Dipendenze ASL Roma 2, Presidente S.I.Pa.D.

\# Direttore Dipartimento di Salute Mentale e delle Dipendenze, ASST Melegnano e della Martesana (Città Metropolitana di Milano), Past President FeDerSerD. perché la maggior parte di essi (il 34.1\% in Italia) ha una storia di consumo di sostanze (dati della Relazione Annuale al Parlamento sullo stato delle Tossicodipendenze, 2017).

Dal punto di vista epidemiologico le Nazioni Unite hanno stimato che al Mondo vi sono circa 12 milioni di consumatori di sostanze che si iniettano droghe per via endovenosa (i cosiddetti PWID - People Who Inject Drugs) (UN World Report, 2016). Di questi circa 6 milioni sono HCV+, mentre I'80\% dei PWID HIV+ è coinfetto da HCV (UN World Report, 2016).

Dati europei evidenziano come il 67\% dei PWID, cioè un numero corrispondente a circa 3 milioni di soggetti, siano anti-HCV+ (Nelson et al., 2011). I dati indicano, inoltre, che in Europa il $49 \%$ dei PWID infetti non è diagnosticato (Wiessing et al., 2014).

L'uso iniettivo di sostanze rappresenta pertanto il fattore di rischio più importante per la trasmissione dell'infezione. Viene inoltre rilevato in reports emessi dall'autorità di tutela della salute mondiale come l'uso per via parenterale di sostanze sia responsabile del 23\% delle nuove infezioni (WHO Global Hepatitis Report, 2017) e come ogni PWID con infezione da HCV sia in grado di infettare almeno 20 altri consumatori, entro i primi 3 anni dall'inizio del contagio (Magiorkinis et al., 2013). Questi dati pertanto documentano come i PWID rappresentino attualmente il maggiore serbatoio della malattia e la principale fonte di infezione. 
Nel 2016 i Ser.D. hanno avuto in carico 143.271 utenti (dati Relazione al Parlamento sullo Stato delle Tossicodipendenze, 2017), mentre si stima che circa il doppio siano i consumatori problematici che non sono in carico ai Servizi, ma che necessiterebbero di un trattamento. In altri termini, in Italia vi sarebbero almeno 450.000 consumatori ad alto rischio, cioè soggetti che a seguito del loro consumo problematico di sostanze potrebbero avere delle serie conseguenze negative (anche in termini infettivologici) sulla propria salute. I dati della relazione al Parlamento del 2017 indicano che dei circa 150.000 soggetti in carico ai Ser.D. almeno il 27\% siano PWID (dato probabilmente sottostimato) e più del $70 \%$ policonsumatori.

L'esatta prevalenza dell'HCV nei consumatori di sostanze in Italia non è nota. Un recente studio di natura epidemiologica condotto su 21 Ser.D. italiani che ha coinvolto 543 consumatori di sostanze ha dimostrato che il $63.9 \%$ degli utenti sono anti-HCV+ (Stroffolini et al., 2012). Secondo questo dato si può ipotizzare che in Italia fra i 150.000 utenti già in trattamento nei Ser.D. almeno 90.000 siano $\mathrm{HCV}+$. Un dato che potrebbe arrivare anche a 270.000 soggetti se si considera che circa 300.000 sono gli utenti ancora non trattati dai Ser.D. e che potrebbero necessitare di una presa in carico.

Allo stesso modo non esistono dati certi sulla prevalenza dell'epatite $\mathrm{C}$ nei detenuti, ma di certo fra essi il comportamento a maggior rischio per il contagio dell'infezione è il consumo di sostanze (in particolare per via endovenosa), seguito dalle pratiche dei tatuaggi e dal sesso non protetto (Zampino et al., 2015). I dati ufficiali del Dipartimento dell'Amministrazione Penitenziaria indicano che nel corso del 2016 sono state ristrette nei 190 Istituti Penitenziari Italiani 101.995 persone, con presenze giornaliere oscillanti tra i 53 ed i 55.000. Una revisione della letteratura sulla prevalenza dell'HCV all'interno delle carceri ha quantificato la prevalenza della infezione in una percentuale compresa fra il $22.4 \%$ e il $38 \%$ dell'intera popolazione detenuta (Zampino et al., 2015). Questi dati indicano che in Italia sia verosimile che nelle carceri transitino annualmente fino a 3035.000 soggetti anti-HCV+.

I Ser.D. devono implementare I'attività di screening e di diagnosi dell'infezione da HCV. I dati della Relazione al Parlamento del 2017 indicano che i Ser.D. hanno testato per HCV nel 2016 solo il $20.5 \%$ dei loro utenti; di questi il $9 \%$ è risultato positivo.

Il test per I'epatite C è disponibile in tutte le carceri in Italia, ma poche sono le realtà che riescono a testare una percentuale significativa di detenuti. Come nel caso dei Ser.D. i motivi più importanti che limitano lo screening sistematico della popolazione detenuta sono di natura logistica ed organizzativa.

I farmaci ad azione diretta (DAA) per il trattamento dell'infezione da HCV rappresentano una enorme opportunità per implementare la presa in carico dei consumatori di sostanze e per migliorare gli outcome del trattamento (EMCDDA, Hepatitic C among drug users in Europe, 2016). Più in generale l'opportunità di avere a disposizione degli strumenti terapeutici in grado di eliminare nel paziente l'infezione da HCV può permettere di sviluppare modelli integrati di trattamento in grado di implementare lo screening, prevenire le reinfezioni attraverso I'incremento delle azioni di riduzione del danno e migliorare la qualità della vita dei pazienti (Giraudon et al., 2016). I DAA, inoltre, possono rappresentare nelle popolazioni a rischio, come i consumatori di sostanze ed i detenuti, uno strumento terapeutico che può offrire una potenziale valenza anche come misura di prevenzione della malattia (Martin et al., 2015; Metzig et al., 2015).

Le popolazioni a rischio come i consumatori di sostanze ed i detenuti con infezione da HCV possono essere trattati con suc- cesso con i DAA. I dati della letteratura oggi indicano che nei PWID il tasso di risposta virologica sostenuta (SVR) con i DAA è del tutto sovrapponibile alla popolazione infetta generale (Bielen et al., 2017), cioè superiore al 98\%.

Oggi in Italia I'accesso alle cure è garantito a tutti i soggetti con $\mathrm{HCV}$, indipendentemente dal grado di severità della malattia (AIFA, Criteri per il trattamento dell'epatite C, 2017).

Nonostante ciò, allo stato attuale risulta ancora difficile raggiungere molti dei soggetti infetti con comportamenti a rischio, proprio quei soggetti che in termini di sanità pubblica dovrebbero rappresentare il target primario per il trattamento della malattia e per permettere il raggiungimento dell'importante obiettivo di eliminazione dell'infezione a partire dal 2030, così come suggerito dall'OMS.

Numerose esperienze cliniche hanno dimostrato che i consumatori di sostanze, insieme ai soggetti con problemi di natura psichiatrica, hanno un elevato rischio di sviluppare una infezione da HCV (Schaefer et al., 2016). Allo stesso tempo esistono anche evidenze che dimostrano come i soggetti con HCV hanno una maggiore probabilità di sviluppare problemi di natura psichiatrica (soprattutto depressione) e di consumo di sostanze (Yarlott et al., 2017; Schaefer et al., 2016). Più in generale le evidenze dimostrano che (Schaefer et al., 2016):

- la co-morbidità psichiatrica ed il consumo di sostanze hanno una prevalenza maggiore nei soggetti con infezione da HCV, piuttosto che nella popolazione generale;

- la morbidità psichiatrica ed il consumo di sostanze sono associate ad un maggiore rischio di contrarre l'infezione da HCV;

- alcuni sintomi di natura psichiatrica (es. depressione, deficit neuropsicologici) e l'uso di sostanze (es. alcol) sono più frequentemente associati con l'infezione da HCV;

- I'infezione cronica da HCV può condurre a grave "distress" psicologico (stigma, ansia, riduzione della qualità della vita);

- I'infezione cronica da HCV ha effetti significativi a livello centrale sulla neurotrasmissione, attraverso i mediatori delI'infiammazione;

- I'HCV può penetrare all'interno del cervello e replicarsi.

L'obiettivo primario per la presa in carico dei soggetti, appartenenti alla popolazione a rischio, con infezione da HCV è lo sviluppo di un modello di trattamento olistico ed integrato in grado, fra l'altro, anche di favorire l'accesso al trattamento (Wolfe et al., 2015).

Le evidenze indicano che lo sviluppo di una relazione "intensa" fra terapeuta e paziente può incrementare in maniera sensibile l'aderenza al trattamento (Rich et al., 2016). La letteratura e l'esperienza clinica hanno infatti dimostrato che i modelli integrati capaci di sviluppare una forte sinergia fra specialisti, tramite la produzione e l'implementazione di procedure e linee guida comuni, possono facilitare l'accesso alle cure per le popolazioni a rischio (Dillon et al., 2016). Del resto numerose sono le evidenze che dimostrano come il trattamento per l'epatite $C$ nelle popolazioni a rischio può facilitare sia l'efficacia della presa in carico che migliorare gli stili di vita, riducendo i comportamenti a rischio come il consumo di sostanze ed anche la commissione di reati (Batchelder et al., 2015).

Uno degli aspetti più critici del trattamento dell'epatite $C$ nelle popolazioni a rischio può essere il tema della reinfezione. Studi compiuti durante l'era interferonica hanno valutato che il rischio di reinfezione nei PWID è basso e corrisponde ad un tasso di 2.4 per 100 soggetti-anno (Aspinall et al., 2013). In questo senso gli studi hanno anche dimostrato che i tassi più bassi sono proprio nelle nazioni del nord-Europa, dove sono più diffuse e meglio applicate le azioni di riduzione del danno (Aspinall et al., 2013). 
In questo senso, le azioni di riduzione del danno hanno dimostrato di minimizzare la probabilità di reinfezione nella popolazione a rischio (Hawk et al., 2017).

La situazione attuale in Italia pone in evidenza come importanti criticità ed ostacoli attualmente impediscono l'accesso alle cure per il trattamento dell'epatite $\mathrm{C}$ per le popolazioni a rischio. Le maggiori sono quelle che riguardano: la bassa percentuale di screening nella popolazione a rischio; la mancanza, in via sistemica, di una organizzazione integrata interdisciplinare per la presa in carico del paziente con HCV costituita da medici specialisti dei Ser.D., medici penitenziari e medici epatologi ed infettivologici (linkage to care); la preoccupazione del rischio di reinfezione (Konerman et al., 2016).

La letteratura e I'esperienza clinica indicano che le azioni principali per rimuovere gli ostacoli che impediscono il trattamento dell'infezione da HCV nei PWID sono quelli di (WHO, Barriers and facilitators to hepatitis $\mathrm{C}$ treatment for people who inject drugs, 2012):

- sviluppare interventi integrati di tipo sanitario e sociale;

- creare interventi multidisciplinari, che contengano elementi di prevenzione, di protezione del paziente e della comunità e di lotta allo stigma;

- facilitare I'accesso al trattamento, con lo sviluppo di interventi di prossimità;

- implementare la costruzione di reti assistenziali sul territorio (in collegamento con le strutture ospedaliere), tali da facilitare la presa in carico e l'accesso per la persona a tutti i bisogni di cura, compresa la terapia sostitutiva;

- costruire programmi di interventi individuali centrati sulla persona.

In questo senso la presa in carico integrata del soggetto a rischio con infezione da HCV può rappresentare una importante sfida per i moderni sistemi di organizzazione sanitaria ed un forte paradigma di integrazione fra ospedale e territorio, tale da permettere una equità di accesso alle cure fra tutti i soggetti infetti da HCV, ma soprattutto per permettere il raggiungimento dell'importante obiettivo di sanità pubblica di eliminazione della malattia entro il 2030.

\section{Gli elementi della strutturazione della presa in carico}

Le esperienze cliniche e le evidenze dalla letteratura indicano che le azioni principali da compiere per la presa in carico della popolazione a rischio con HCV sono:

- lo screening;

- il referral;

- il trattamento;

- la prevenzione della reinfezione (tramite le azioni di riduzione del danno). In questo senso appare prioritario, in base alle diverse indicazioni fornite dalla letteratura e dall'esperienza clinica, proporre degli algoritmi per la presa in carico della popolazione a rischio con HCV in modo da facilitare la costruzione sul territorio di reti e modelli organizzativi efficaci ed efficienti.

\section{Algoritmi per la presa in carico della popolazione a rischio con $\mathrm{HCV}$}

La fase dello screening (Fig. 1) deve essere caratterizzata dai seguenti elementi essenziali che facilitano l'esecuzione del test da parte del paziente e che consistono nella capacità dei Ser.D. e delle Carceri:

- di eseguire prelievi per diagnosi convenzionale della malattia, eventualmente anche con l'utilizzo di test rapidi;

- di informazione sulla malattia, sui trattamenti e sulle modalità di contagio (tramite brochure, siti web, campagne pubbliche di informazione anche sui social networks);

- di fornire all'utente consueling psicoeducazionale e motivazionale in grado di ottimizzare al massimo l'aderenza dei pazienti al test;

- di offrire sistemi organizzativi in grado di garantire all'utenza l'accesso e l'esecuzione del test sia in entrata che periodicamente durante la presa in carico, in modo da poter intercettare negli ambiti di cura anche eventuali nuove infezioni o re-infezioni.

Fig. 1 - Fase dello screening (Ser.D. e carceri)

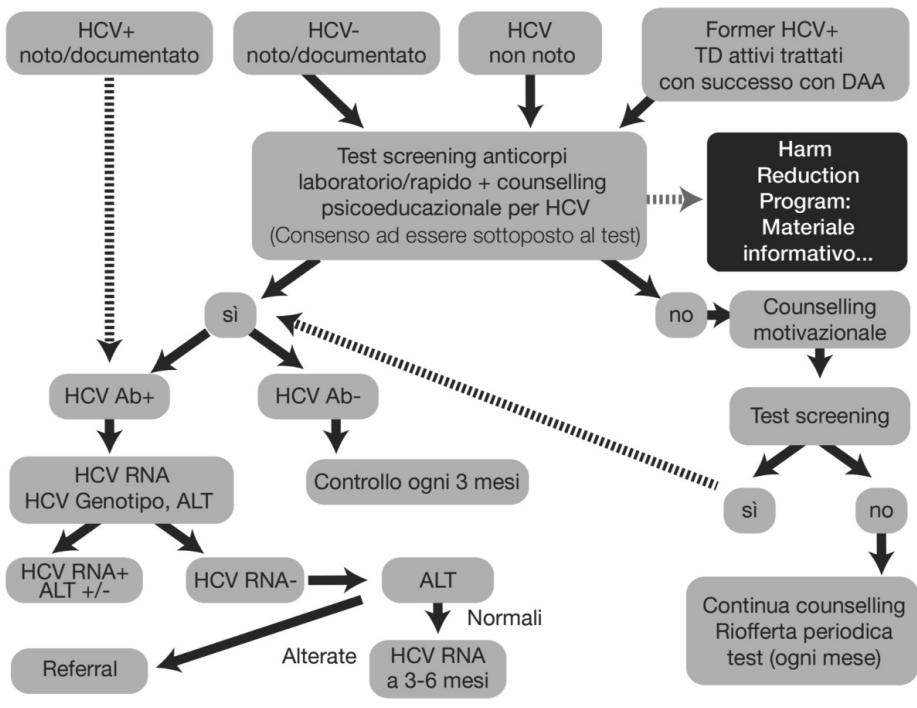

- lo screening per HCV deve essere proposto a tutti i consumatori di sostanze;

- la proposta dello screening deve essere accompagnata da un counseling psicoeducazionale e motivazionale;

- la proposta dello screening deve essere associata alla distribuzione di materiale informativo sulla malattia, sui trattamenti e sulle modalità di contagio;

- lo screening se negativo deve essere riproposto periodicamente (almeno ogni 3 mesi);

- lo screening se rifiutato deve essere riproposto periodicamente abbinato ad un counseling motivazionale (almeno ogni mese):

- lo screening deve essere proposto ai consumatori attivi trattati con successo con DAA per la diagnosi precoce della reinfezione (almeno ogni 3 mesi).

La fase del referral (Fig. 2) è caratterizzata dai seguenti elementi essenziali che hanno l'obiettivo di motivare il paziente al trattamento e di facilitare il suo contatto con lo specialista epatologoinfettivologo che sono:

- la motivazione del paziente al trattamento, attraverso tecniche di colloquio motivazionale;

- il trasferimento al paziente dei principi di base di riduzione del danno (in modo da limitare le conseguenze della malattia sia per sé che per la comunità, anche in termini di prevenzione della reinfezione);

- lo sviluppo di modelli organizzativi che prevedano il referral, preferibilmente nello stesso luogo di presa in carico dell'utente (Ser.D. o Carcere); 
Fig. 2 - Fase di referral (Ser.D. e carceri)

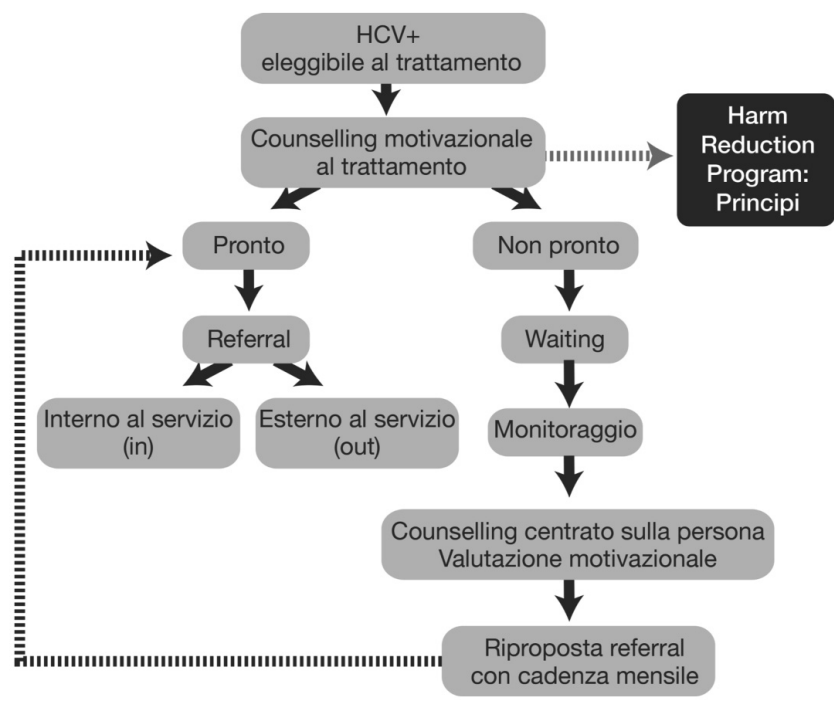

- il trasferimento del paziente allo specialista esclusivamente quando "pronto" al trattamento dal punto di vista motivazionale (mentre il paziente ancora "non pronto" dovrà essere tenuto agganciato al Servizio mediante tecniche di colloquio motivazionale finalizzate alla informazione e allo sviluppo della relazione);

- la proposta periodica per il paziente dell'invio allo specialista per coloro che hanno rifiutato il trattamento;

- tutti i pazienti eleggibili al trattamento devono essere inviati allo specialista (infettivologo/epatologo);

- il referral deve essere accompagnato da un counseling motivazionale

- tutti i pazienti che vengono inviati allo specialista (infettivologo/epatologo) per il trattamento devono ricevere un pro- gramma strutturato di riduzione del danno (conoscenza dei principi e delle misure così come indicate dall'OMS).

La fase del trattamento (Fig. 3) è caratterizzata dai seguenti elementi essenziali che hanno l'obiettivo di trattare in maniera appropriata il paziente attraverso:

- una completa valutazione clinica compiuta dai medici epatologi-infettivologi finalizzata all'inizio del trattamento (preferibilmente nel luogo di presa in carico dell'utente);

- un attento monitoraggio (congiunto fra specialisti) della compliance al trattamento basata sulla relazione terapeutica e sul supporto motivazionale;

- un controllo della risposta virologica 12 settimane dalla fine del trattamento e monitoraggio periodico a 3-6 mesi dalla fine del trattamento per valutare l'eventuale reinfezione da parte degli specialisti (singola e/o congiunta) per gli obiettivi di risposta tossicologica e di valutazione della qualità della vita;

- una offerta di programmi di riduzione del danno, finalizzati alla riduzione dei comportamenti a rischio, attraverso lo sviluppo di training di abilità nella gestione delle azioni di riduzione del danno e la fornitura di kit di harm reduction;

- un momento di verifica delle capacità del paziente di utilizzo delle misure di riduzione del danno;

- tutti i pazienti HCV RNA + possono essere eleggibili al trattamento;

- tutti i pazienti che presentano i criteri clinici per l'inizio del trattamento devono essere trattati con i DAA;

- tutti i pazienti devono ricevere un programma strutturato di riduzione del danno (con training di abilità);

- il monitoraggio del trattamento deve prevedere la valutazione dell'aderenza alla terapia e la valutazione del raggiungimento degli outcome infettivologici, tossicologici e comportamentali

Fig. 3 - Fase del trattamento (Epatologi-Infettivologi + Ser.D. e carceri)

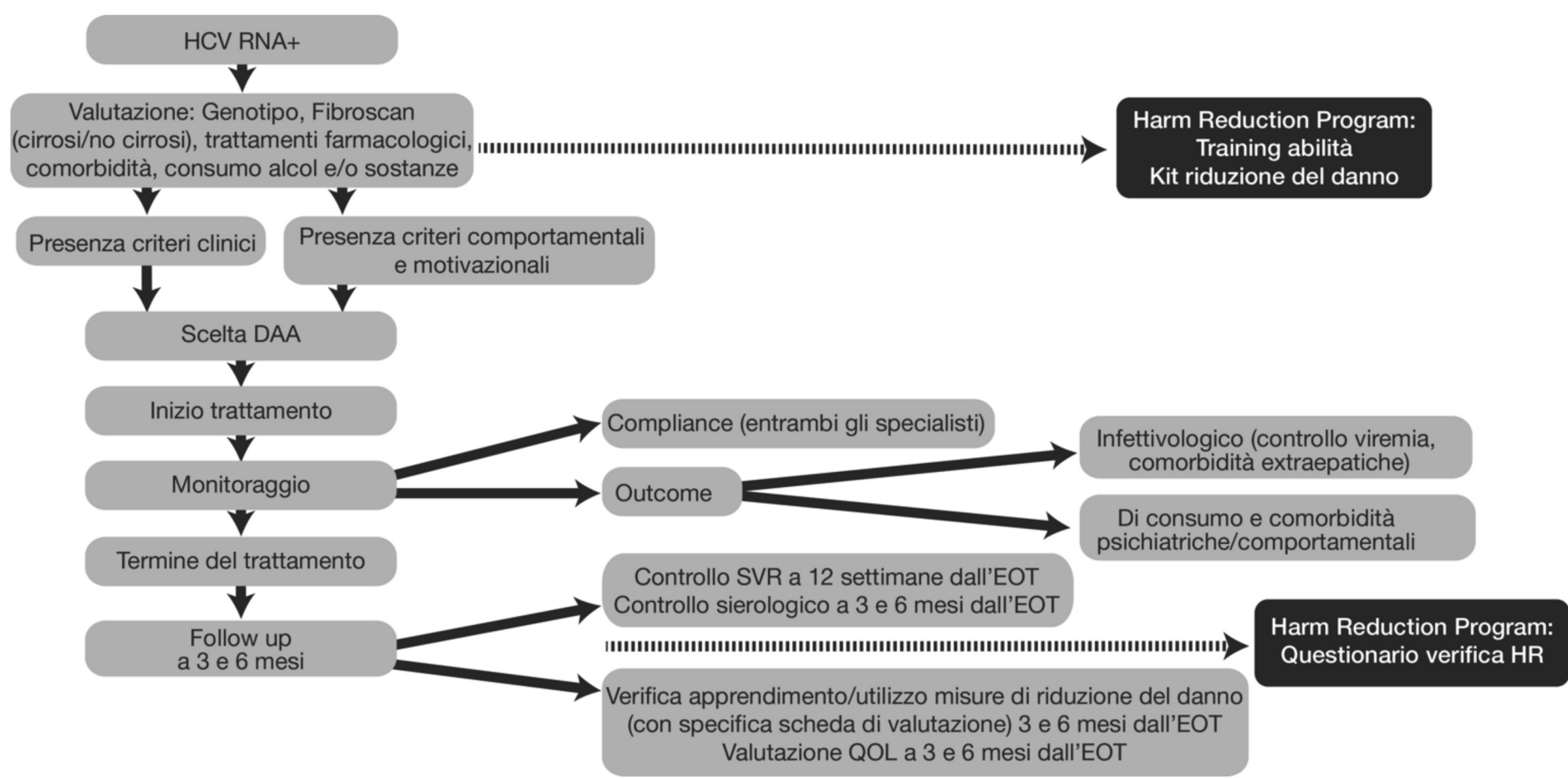


- il termine del trattamento deve essere seguito da un follow up di tipo infettivologico e da una valutazione sulle capacità di utilizzo delle misure di riduzione del danno;

- tutti i pazienti che vengono trattati con i DAA devono ricevere un kit di riduzione del danno.

\section{Riduzione del danno}

La prevenzione dell'infezione è un aspetto di primaria importanza nella presa in carico della popolazione a rischio con HCV ed è un elemento che deve essere sempre associato al trattamento farmacologico.

La prevenzione dell'infezione deve avvenire attraverso le azioni di riduzione del danno, così come sono state individuate dall'OMS.

In particolare, le azioni di riduzione del danno prevedono l'utilizzo di:

- materiale informativo e di supporto (anche su web) (da utilizzare in via prioritaria nelle fasi di screening);

- training specifici sui principi di base di riduzione del danno (da utilizzare in via prioritaria nelle fasi di referral);

- training di abilità sulle azioni di riduzione del danno (da utilizzare in via prioritaria nelle fasi di trattamento);

- kit di riduzione del danno da fornire alla popolazione a rischio (da utilizzare in via prioritaria nelle fasi di trattamento);

- processi di verifica di acquisizione/utilizzo delle misure di riduzione del danno (da utilizzare in via prioritaria nelle fasi di follow-up).

Le azioni di riduzione del danno si dovranno articolare seguendo tutte le fasi della "filiera" di presa in carico (Fig. 4) con lo scopo di rafforzare il raggiungimento degli obiettivi previsti dalle singole fasi, facilitare l'accesso e l'aderenza al trattamento ma, soprattutto, riducendo i comportamenti a rischio e minimizzando il tasso di reinfezione.

- Le misure di riduzione del danno, come suggerite dalI'OMS, devono essere applicate a tutti i consumatori di sostanze (indipendentemente se attivi e/o in trattamento con DAA);

- le misure di riduzione del danno devono accompagnare, con azioni e modalità specifiche, tutte le fasi della presa in carico del consumatore di sostanze con infezione da HCV (screening - referral - trattamento - follow up);

- kit di riduzione del danno devono essere forniti a tutti i consumatori di sostanze che sono (o sono stati) in trattamento con i DAA.

\section{I principi fondamentali per la presa in carico del consumatore di sostanze e del detenuto con $\mathrm{HCV}$}

Il trattamento della popolazione a rischio (consumatori di sostanze e detenuti) deve diventare una priorità per i sistemi sanitari sia per garantire l'equità di accesso alle cure che per raggiungere l'obiettivo di sanità pubblica di eliminazione dell'HCV. I programmi di presa in carico devono essere integrati, multidisciplinari flessibili, individuali e di prossimità.

I programmi di presa in carico devono essere basati sulle evidenze scientifiche e devono essere diffusi omogeneamente su tutto il territorio nazionale.
Fig. 4 - Riduzione del danno

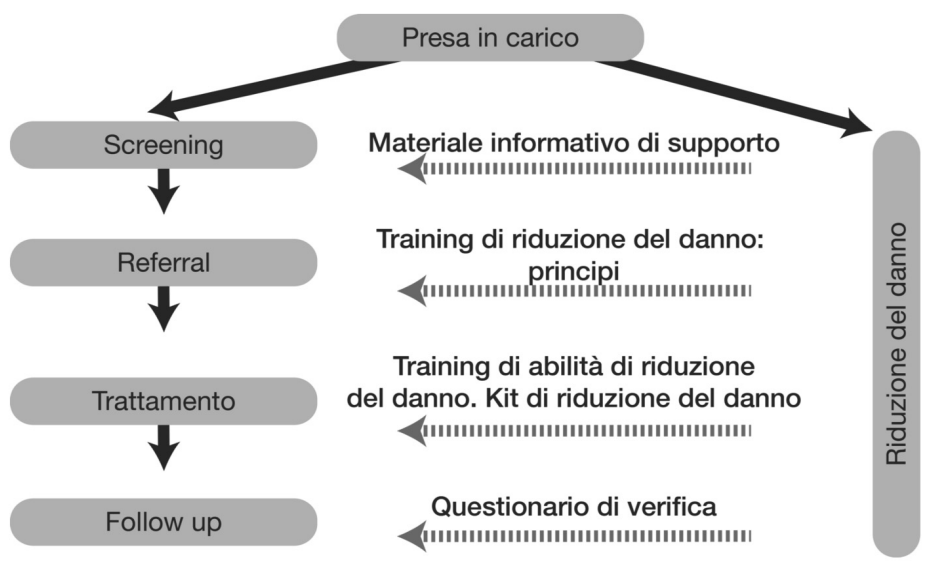

La presa in carico deve essere supportata da procedure e linee guida che devono comprendere anche le azioni di riduzione del danno, cosi come suggerite dall'OMS.

\section{Bibliografia}

AIFA (2017). Criteri di trattamento per l'epatite C. -- www.agenzia farmaco.gov.it/content/aggiornamento-epatite-c.

Aspinall E.J., Corson S., Dovle J.S., Grebely J., Hutchinson S.J., Dore G.J., Goldberg D.J., Hellard M.E. (2013). Treatment of hepatitis C virus infection among people who are actively injecting drugs: a systematic review and meta-analysis. Clin. Infect. Dis., 57(Suppl 2): S80-9.

Batchelder A.W., Peyser D., Nahvi S., Arnsten J.H., Litwin A.H. (2015). Hepatitis C treatment turned me around: "Psychological and behavioral transformation related to hepatitis C treatment". Drug Alcohol Depend., 153: 66-71.

Bielen R., Moreno C., Van Vlierberghe H., Bourgeois S., Mulkay J.P., Vanwolleghem T., Verlinden W., Brixko C., Decaestecker J., De Galocsy C., Janssens F., Cool M., Van Steenkiste C., D'heygere F., Cools W., Nevens F., Robaeys G. (2017). Belgian experience with direct acting antivirals in people who inject drugs. Drug Alcohol Depend., 177: 214-220.

Dillon J.F., Lazarus J.V., Razavi H.A. (2016). Urgent action to fight hepatitis $\mathrm{C}$ in people who inject drugs in Europe. Hepatology Medicine and Policy, 1: 2.

European Monitoring Centre for Drug and Drug Addiction (EMCDDA) European $\mathrm{C}$ among drug users in Europe, epidemiology, treatment and prevention (2016). www.emcdda.europa.eu/system/files/ publications/2953/TDXD16002ENN_final_web.pdf.

Hawk M., Coulter R.W.S., Egan J.E., Fisk S., Reuel Friedman M., Tula M., Kinsky S. (2017). Harm reduction principles for healthcare settings. Harm Reduct. J., 14(1): 70.

Konerman M.A., Lok A.S.F. (2016). Hepatitis C treatment and barriers to eradication. Clin. Transl. Gastroenterology, 7: e193.

Magiorkinis G., Sypsa V., Magiorkinis E., Paraskevis D., Katsoulidou A., Belshaw R., Fraser C., Pybus O.G., Hatzakis A. (2013). Integrating phylodynamics and epidemiology to estimate transmission diversity in viral epidemics. PLoS Comput Biol., 9(1): e1002876.

Martin N.K., Vickerman P., Dore G., Hickman M. (2015). The HCV epidemics in key populations (including PWID, prisoners, and MSM): the use of DAAs as treatment for prevention. Curr Opin HIV AIDS, 10: 374-380.

Metzig C., Surey J., Francis M., Conneely J., Abubakar I., White P.J. (2017). Impact of hepatitis C treatment as prevention for people who inject drug is sensitive to contact network structure. Sci Rep. 2017, May 12, 7(1): 1833. 
Nelson P.K., Mathers B.M., Cowie B., Hagan H., Des Jarlais D., Horyniak D., Degenhardt L. (2011). Global epidemiology of hepatitis $B$ and hepatitis C in people who inject drugs: results of systematic reviews. Lancet, 378(9791): 571-83.

Relazione Annuale al Parlamento sullo Stato delle Tossicodipendenze, anno 2017, www.politicheantidroga.gov.it/media/2153/relazione-alparlamento_2017.pdf.

Rich Z.C., Chu C., Mao J., Zhou K., Cai W., Ma Q., Volberding P., Tucker J.D. (2016). Facilitators of HCV treatment adherence among people who inject drugs: a systematic qualitative review and implications for scale up of direct acting antivirals. BMC Public Health, 16: 994.

Schaefer M., Capuron L., Friebe A., Diez-Quevedo C., obaeys G., Neri S., Foster G.R., Kautz A., Forton D., Pariante C.M. (2012). Hepatitis C infection, antiviral treatment and mental health: a European expert consensus statement. J. Hepathol., 57: 1379-1390.

Stroffolini T., D'Egidio P.F., Aceti A., Filippini P., Puoti M., Leonardi C., Almasio P.L., DAVIS Drug Addicted, HCV Prevalence in Italy an Epidemiological, Observational, Cross-Sectional, Multicenter Study Participating Centers (2012). Hepatitis C virus infection among drug addicts in Italy. J. Med. Virol., 84: 1608-1612.

UN World Report (2016). www.unodc.org/doc/wdr2016/WORLD_ DRUG_REPORT_2016_web.pdf.
Yarlott L., Heald E., Forton D. (2017). Hepatitis C virus infection, and neurological and psychiatric disorder - a review. J. Adv. Res., 8: 138-148.

World Health Organization, Barriers and facilitators to hepatitis C treatment for people who inject drugs, a qualitative study (2012). www.euro.who.int/_data/assets/pdf_file/0011/179750/Barriers-andfacilitators-to-hepatitis-C-treatment-for-PWID-A-qualitative-studyJune-2012-rev-5.pdf?ua=1.

WHO Global Hepatitis Report (2017). http://apps.who.int/iris/ bitstream/10665/255016/1/9789241565455-eng.pdf?ua=1.

Wiessing L., Ferri M., Grady B., Kantzanou M., Sperle I., Cullen K.J., EMCDDA DRID group, Hatzakis A., Prins M., Vickerman P., Lazarus J.V., Hope V.D., Mathei C. (2014). PLoS One, 28, 9(7): e103345.

Wolfe D., Luhmann N., Harris M., Momenghalibaf A., Albers E., Byrne E., Swan T. (2015). Human rights to access to hepatitis $C$ treatment for people who inject drugs. Inter. J. Drug Policy, 26: 1072-1080.

Zampino R., Coppola N., Sagnelli C., Di Caprio G., Sagnelli E. (2015). Hepatitis $C$ virus infection and prisoners: epidemiology, outcome and treatment. World J Hepatol., 7(21): 2323-30.

Pubblicazione realizzata con il contributo non condizionante di Gilead Sciences 